\title{
Multiple Sklerose - zwischen Grundlagenforschung und klinischer Anwendung
}

$\mathrm{D}$ ie molekulare Grundlagenforschung des letzten Jahrzehnts hat uns deutliche Fortschritte für das Verständnis der Pathogenese der Multiplen Sklerose (MS) ermöglicht. Wir gehen davon aus, dass es sich bei der Multiplen Sklerose um ein heterogenes Syndrom handelt, bei dem neben genetischen Faktoren auch Umwelteinflüsse und Prägung des Immunsystems eine Rolle spielen. Diese Heterogenität der Multiplen Sklerose spiegelt sich bereits in unterschiedlichen Typen akuter MSPlaques wider. Darüber hinaus liefert sie auch eine Erklärung dafür, dass manche Patienten auf die modernen Immuntherapien sehr gut ansprechen, während andere Patienten leider progressiv schlechter werden.

Mit diesem Schwerpunktheft haben wir uns die Aufgabe gestellt, aus dem angeführten aktuellen Forschungsfeld relevante Themen prägnant abzuhandeln. Aus dem Institut für Neuroimmunologie, Charité Berlin, werden innovative experimentelle Arbeiten zu Mechanismen neuronalen Zelltods bei der Multiplen Sklerose diskutiert. Sie zeigen uns, welche neuartigen Schädigungsprinzipien und damit assoziiert auch molekulare Therapien zukünftig diesen Aspekt abdecken können. Am Institut für Neuropathologie der Universität Göttingen werden die Fortschritte der humanen molekularen Neuropathologie intensiv erforscht. Diese betreffen den frühen axonalen Schaden, die möglichen Mechanismen chronischer Progredienz mit axonaler Schädigung sowie vor allem die in den letzten beiden Jahren beschriebenen kortikalen Läsionen. Ein besseres Verständnis dieser Vorgänge ermöglicht vor allem die Therapie von kognitiven Störungen in späteren Krankheitsverläufen. Aus der Neurologischen Universitätsklinik, Sankt Josef-Hospital Bochum werden Mechanismen und Prinzipien der Immuntherapie im klinischen Alltag zusammengestellt. Dies soll eine möglichst individualisierte Therapie der MS erleichtern. Schließlich geben meine eigenen Mitarbeiter einen Überblick über zukünftige Therapieentwicklungen: welche Medikamente stehen in der Zulassungs-Pipeline, und welche werden momentan aus dem experimentellen Bereich übertragen? All dies belegt, dass eine erfolgreiche Therapie der MS nur unter Einbeziehung verschiedener immunologischer und neurobiologischer Aspekte möglich sein wird. Dies ist bisher in Deutschland nur im Institut für MS Forschung verwirklicht, an dem drei der vier Autorengruppen mitarbeiten.

Wir hoffen, dass wir mit der Auswahl der Themen dieses Hefts den interessierten Kollegen aktuelle Informationen liefern können, die ein Bindeglied zwischen moderner Grundlagenforschung und klinischer Anwendung im Sinne „translationaler Medizin“ darstellen.

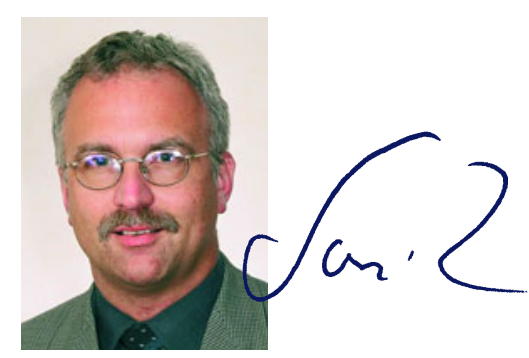

Prof. Ralf Gold, Göttingen 